\title{
The Existence Result for a Class of p-Kirchhoff-Type Problem with a Multilinear Growth Nonlinearity
}

\author{
Jiangyan Yao and Wei Han \\ Department of Mathematics, North University of China, Taiyuan, Shanxi 030051, China \\ Correspondence should be addressed to Wei Han; sh_hanweiweil@126.com
}

Received 11 April 2019; Accepted 17 June 2019; Published 7 July 2019

Academic Editor: Marek Galewski

Copyright (C) 2019 Jiangyan Yao and Wei Han. This is an open access article distributed under the Creative Commons Attribution License, which permits unrestricted use, distribution, and reproduction in any medium, provided the original work is properly cited.

In this paper, we firstly discuss the existence of the least energy sign-changing solutions for a class of p-Kirchhoff-type problems with a $(2 p-1)$-linear growth nonlinearity. The quantitative deformation lemma and Non-Nehari manifold method are used in the paper to prove the main results. Remarkably, we use a new method to verify that $\mathscr{M}_{b} \neq \emptyset$. The main results of our paper are the existence of the least energy sign-changing solution and its corresponding energy doubling property. Moreover, we also give the convergence property of the least energy sign-changing solution as the parameter $b \searrow 0$.

\section{Introduction and the Main Results}

In this paper, we are devoted to investigating the existence of the least energy sign-changing solutions for the following p-Kirchhoff-type problem with a (2p-1)-linear growth nonlinearity:

$$
\begin{aligned}
-\left(a+b \int_{\Omega}|\nabla u|^{p} d x\right) \Delta_{p} u & =\lambda|u|^{p-2} u+|u|^{2 p-2} u, \\
x \in \Omega, & \\
u & =0, \quad x \in \partial \Omega,
\end{aligned}
$$

where $\Omega$ is a bounded domain in $R^{N}(N=1,2,3), a, b>0$, $\lambda<a \lambda_{1}, \lambda_{1}$ is the first eigenvalue of the following problem:

$$
\begin{aligned}
-\Delta_{p} \phi & =\lambda|\phi|^{p-2} \phi, \quad x \in \Omega, \\
\phi & =0, \quad x \in \partial \Omega .
\end{aligned}
$$

In fact, the related problems have been studied extensively, especially on the existence of the positive solutions, multiple solutions, ground state solutions, and least energy sign-changing solutions. In [1], Li and Sun studied the existence and multiplicity of solutions for the Kirchhoff equations with asymptotically linear nonlinearities; the mountain pass theorem was used in the paper. Guo, Ma, and Zhang [2] studied a class of autonomous Kirchhoff-type equation. By a simple transformation, they found that the solutions of autonomous Kirchhoff-type equation or system could be obtained by using the known solutions of the corresponding local equation or system, which is very interesting. In [3], Ying Li and Lin Li considered the existence and multiplicity of solutions to a class of $\mathrm{p}(\mathrm{x})$-Laplacian-like equations. They introduced a revised Ambrosetti-Rabinowitz condition and obtained that the problem had a nontrivial solution and infinitely many solutions, respectively. Meanwhile, in [4], Luca Vilasi proved an eigenvalue theorem for a stationary $\mathrm{p}(\mathrm{x})$-Kirchhoff problem by using variational techniques, and the author also provided an estimate for the range of such eigenvalues. For more details, we refer the reader to [5-30].

In $[31,32]$, the authors studied the following Kirchhofftype problems in bounded domains:

$$
\begin{aligned}
-\left(a+b \int_{\Omega}|\nabla u|^{2}\right) \Delta u & =f(u), \quad x \in \Omega, \\
u & =0, \quad x \in \partial \Omega,
\end{aligned}
$$

under different assumptions on $f(u)$, the authors mainly use the quantitative deformation lemma and the degree theory to get the existence of the least energy sign-changing solution 
and its corresponding convergence property as the parameter $b \searrow 0$. From the assumptions on $f(u)$, we can easily find that both in $[31,32] f(u)$ satisfies 3 -superlinear growth at infinity and superlinear growth at zero.

Later, some scholars made some expanding work; we can find some details in [33]. In [33], we know that the nonlinearity $f$ satisfies $(2 p-1)$-superlinear growth condition at infinity.

Motivated by the above works, a natural question is that if there exists a ground state sign-changing solution for problem (1). However, up to now, no paper has appeared in the literature which discusses the existence and convergence property of the solution for the p-Kirchhoff-type problem with a $(2 p-1)$-linear growth nonlinearity. This paper attempts to fill this gap in the literature.

Throughout this paper, we will make full use of the following notations. Let $W=W_{0}^{1, p}(\Omega)$ be the usual Sobolev space equipped with the following norm:

$$
\|u\|=\left(\int_{\Omega}|\nabla u|^{p} d x\right)^{1 / p} .
$$

$\|\cdot\|_{s}$ denotes the usual Lebesgue space $L^{s}(\Omega)$ norm. $S$ is the best Sobolev constant for the embedding of $W_{0}^{1, p}(\Omega)$ in $L^{2 p}(\Omega)$; that is,

$$
\|u\|_{2 p} \leq S^{-1 / p}\|u\| .
$$

From the above definition, we give the energy functional corresponding to problem (1) by

$$
\begin{aligned}
I_{b}(u)= & \frac{a}{p} \int_{\Omega}|\nabla u|^{p} d x+\frac{b}{2 p}\left(\int_{\Omega}|\nabla u|^{p} d x\right)^{2} \\
& -\frac{\lambda}{p} \int_{\Omega}|u|^{p} d x-\frac{1}{2 p} \int_{\Omega}|u|^{2 p} d x .
\end{aligned}
$$

Clearly, $I_{b}$ is well defined on $W$ and is of $C^{1}$ class. For each $u, v \in W$, by a simple calculation, we have

$$
\begin{aligned}
& \left\langle I_{b}^{\prime}(u), v\right\rangle \\
& =\left(a+b \int_{\Omega}|\nabla u|^{p} d x\right) \int_{\Omega}|\nabla u|^{p-2} \nabla u \cdot \nabla v d x \\
& \quad-\int_{\Omega} \lambda|u|^{p-2} u v d x-\int_{\Omega}|u|^{2 p-2} u v d x .
\end{aligned}
$$

Obviously, the critical points of $I_{b}$ are corresponding to the weak solutions of problem (1). If $u \in W$ is a sign-changing solution of problem (1), then

(i) $u$ is a solution of problem (1), that is, $u$ is a critical point of $I_{b}$;

(ii) $u^{ \pm} \neq 0$, where $u^{+}=\max \{u(x), 0\}, u^{-}=\min \{u(x), 0\}$.

For $u=u^{+}+u^{-}$, from (6) and (7), we have

$$
\begin{aligned}
I_{b}(u) & =I_{b}\left(u^{+}\right)+I_{b}\left(u^{-}\right)+\frac{b}{p}\left\|\nabla u^{+}\right\|_{p}^{p}\left\|\nabla u^{-}\right\|_{p}^{p} ; \\
\left\langle I_{b}^{\prime}(u), u^{+}\right\rangle & =\left\langle I_{b}^{\prime}\left(u^{+}\right), u^{+}\right\rangle+b\left\|\nabla u^{+}\right\|_{p}^{p}\left\|\nabla u^{-}\right\|_{p}^{p} ; \\
\left\langle I_{b}^{\prime}(u), u^{-}\right\rangle & =\left\langle I_{b}^{\prime}\left(u^{-}\right), u^{-}\right\rangle+b\left\|\nabla u^{+}\right\|_{p}^{p}\left\|\nabla u^{-}\right\|_{p}^{p}
\end{aligned}
$$

When $b=0$, problem (1) reduces to the following problem:

$$
\begin{aligned}
-a \Delta_{p} u & =\lambda|u|^{p-2} u+|u|^{2 p-2} u, \quad x \in \Omega, \\
u & =0, \quad x \in \partial \Omega .
\end{aligned}
$$

The corresponding energy functional $I_{0}: W \longrightarrow R$ is defined by

$$
\begin{aligned}
I_{0}(u)= & \frac{a}{p} \int_{\Omega}|\nabla u|^{p} d x-\frac{\lambda}{p} \int_{\Omega}|u|^{p} d x \\
& -\frac{1}{2 p} \int_{\Omega}|u|^{2 p} d x .
\end{aligned}
$$

Also, we can compute that

$$
\begin{aligned}
\left\langle I_{0}^{\prime}(u), v\right\rangle= & \int_{\Omega} a|\nabla u|^{p-2} \nabla u \cdot \nabla v d x \\
& -\int_{\Omega} \lambda|u|^{p-2} u v d x-\int_{\Omega}|u|^{2 p-2} u v d x .
\end{aligned}
$$

For $b>0$, problem (1) is called a nonlocal problem since the appearance of the nonlocal term $\left(\int_{\Omega}|\nabla u|^{p} d x\right) \Delta_{p} u$. The differences posed by the nonlocal term make the method in solving problem (11) cannot be applied to solve problem (1), which makes the study of our paper very interesting and meaningful.

In our paper, we restrict $u$ in the following sets to find the ground state sign-changing solutions of (1) and (11),

$$
\begin{aligned}
\mathscr{M}_{b} & =\left\{u \in W: u^{ \pm} \neq 0,\left\langle I_{b}^{\prime}(u), u^{+}\right\rangle=\left\langle I_{b}^{\prime}(u), u^{-}\right\rangle\right. \\
& =0\}, \\
\mathscr{M}_{0} & =\left\{u \in W: u^{ \pm} \neq 0,\left\langle I_{0}^{\prime}(u), u^{+}\right\rangle=\left\langle I_{0}^{\prime}(u), u^{-}\right\rangle\right. \\
& =0\},
\end{aligned}
$$

and we define $m_{b}=\inf _{u \in \mathscr{M}_{b}} I_{b}(u)$ and $m_{0}=\inf _{u \in \mathscr{M}_{0}} I_{0}(u)$.

To get the ground state solutions, we define the following sets:

$$
\begin{aligned}
& \mathcal{N}_{b}=\left\{u \in W: u \neq 0,\left\langle I_{b}^{\prime}(u), u\right\rangle=0\right\}, \\
& \mathcal{N}_{0}=\left\{u \in W: u \neq 0,\left\langle I_{0}^{\prime}(u), u\right\rangle=0\right\}
\end{aligned}
$$

and consider the following minimization problem:

$$
\begin{aligned}
& c_{b}=\inf _{u \in \mathscr{N}_{b}} I_{b}(u), \\
& c_{0}=\inf _{u \in \mathcal{N}_{0}} I_{0}(u) .
\end{aligned}
$$

Since $\mathscr{M}_{b} \subset \mathcal{N}_{b}$, we can immediately get $m_{b} \geq c_{b}$. The main results of the paper are described as follows.

Theorem 1. For $b \in\left(0,1 / 2 S^{2}\right)$ and $\lambda<a \lambda_{1}$, problem (1) has at least one ground state sign-changing solution, which precisely has two nodal domains. Moreover, $m_{b}>2 c_{b}$. 
Theorem 2. For each $\lambda<a \lambda_{1}$, for any sequence $\left\{b_{n}\right\}$ small enough with $b_{n} \searrow 0$ as $n \longrightarrow \infty$, there exists a subsequence still denoted by $\left\{b_{n}\right\}$, such that $u_{b_{n}}$ convergent to $u_{0}$ strongly in $W_{0}^{1, p}(\Omega)$, where $u_{0}$ is a ground state sign-changing solution of problem (11), which changes sign only once.

Our paper is organized as follows. In Section 2, some preliminary lemmas are given to prove the main results. In Sections 3 and 4, we are devoted to proving the main results of the paper.

\section{Some Critical Preliminaries}

The following several lemmas are crucial to prove our main results.

Lemma 3. If $b>0, \lambda<a \lambda_{1}, u \in W$ satisfies $u^{ \pm} \neq 0$ and

$$
\begin{aligned}
& b\left\|\nabla u^{+}\right\|_{p}^{2 p}+b\left\|\nabla u^{+}\right\|_{p}^{p}\left\|\nabla u^{-}\right\|_{p}^{p}<\int_{\Omega}\left|u^{+}\right|^{2 p} d x, \\
& b\left\|\nabla u^{-}\right\|_{p}^{2 p}+b\left\|\nabla u^{+}\right\|_{p}^{p}\left\|\nabla u^{-}\right\|_{p}^{p}<\int_{\Omega}\left|u^{-}\right|^{2 p} d x,
\end{aligned}
$$

then there exists a unique pair $\left(s_{u}, t_{u}\right)$ of positive numbers such that

(i) $s_{u} u^{+}+t_{u} u^{-} \in \mathscr{M}_{b}$;

(ii) $I_{b}\left(s_{u} u^{+}+t_{u} u^{-}\right)=\max _{s, t \geq 0} I_{b}\left(s u^{+}+t u^{-}\right)$.

Proof. (i) If $s u^{+}+t u^{-} \in \mathscr{M}_{b}$, then from (7), (9), and (10), we have

$$
\begin{aligned}
& \left\langle I_{b}^{\prime}\left(s u^{+}+t u^{-}\right), s u^{+}\right\rangle \\
& =a s^{p}\left\|\nabla u^{+}\right\|_{p}^{p}+b s^{2 p}\left\|\nabla u^{+}\right\|_{p}^{2 p} \\
& \quad+b s^{p} t^{p}\left\|\nabla u^{+}\right\|_{p}^{p}\left\|\nabla u^{-}\right\|_{p}^{p}-s^{p} \int_{\Omega} \lambda\left|u^{+}\right|^{p} d x \\
& \quad-s^{2 p} \int_{\Omega}\left|u^{+}\right|^{2 p} d x=0
\end{aligned}
$$

and

$$
\begin{aligned}
& \left\langle I_{b}^{\prime}\left(s u^{+}+t u^{-}\right), t u^{-}\right\rangle \\
& =a t^{p}\left\|\nabla u^{-}\right\|_{p}^{p}+b t^{2 p}\left\|\nabla u^{-}\right\|_{p}^{2 p} \\
& \quad+b s^{p} t^{p}\left\|\nabla u^{+}\right\|_{p}^{p}\left\|\nabla u^{-}\right\|_{p}^{p}-t^{p} \int_{\Omega} \lambda\left|u^{-}\right|^{p} d x \\
& \quad-t^{2 p} \int_{\Omega}\left|u^{-}\right|^{2 p} d x=0 .
\end{aligned}
$$

Let $S=s^{p}$ and $T=t^{p}$, the above equations correspond to the following system:

$$
\begin{aligned}
& S \int_{\Omega}\left|u^{+}\right|^{2 p} d x-b S\left\|\nabla u^{+}\right\|_{p}^{2 p}-b T\left\|\nabla u^{+}\right\|_{p}^{p}\left\|\nabla u^{-}\right\|_{p}^{p} \\
& \quad=a\left\|\nabla u^{+}\right\|_{p}^{p}-\int_{\Omega} \lambda\left|u^{+}\right|^{p} d x
\end{aligned}
$$

$$
\begin{aligned}
& T \int_{\Omega}\left|u^{-}\right|^{2 p} d x-b T\left\|\nabla u^{-}\right\|_{p}^{2 p}-b S\left\|\nabla u^{+}\right\|_{p}^{p}\left\|\nabla u^{-}\right\|_{p}^{p} \\
& \quad=a\left\|\nabla u^{-}\right\|_{p}^{p}-\int_{\Omega} \lambda\left|u^{-}\right|^{p} d x .
\end{aligned}
$$

Obviously, if we can prove that system (20) has the unique solution $(S, T)$, then $\left(s=S^{1 / p}, t=T^{1 / p}\right)$ is the unique solution for (18) and (19).

Let

$$
\begin{aligned}
& D \\
& =\left.\left|\begin{array}{ll}
\int_{\Omega}\left|u^{+}\right|^{2 p} d x-b\left\|\nabla u^{+}\right\|_{p}^{2 p} & -b\left\|\nabla u^{+}\right\|_{p}^{p}\left\|\nabla u^{-}\right\|_{p}^{p} \\
& -b\left\|\nabla u^{+}\right\|_{p}^{p}\left\|\nabla u^{-}\right\|_{p}^{p}
\end{array} \int_{\Omega}\right| u^{-}\right|^{2 p} d x-b\left\|\nabla u^{-}\right\|_{p}^{2 p} \mid \\
& >0 .
\end{aligned}
$$

For $\lambda<a \lambda_{1}$, we have $a\left\|\nabla u^{ \pm}\right\|_{p}^{p}>\lambda \int_{\Omega}\left|u^{ \pm}\right|^{p}$. Since $\int_{\Omega}\left|u^{-}\right|^{2 p}-$ $b\left\|\nabla u^{-}\right\|_{p}^{2 p}>0$, then

$$
\begin{aligned}
& D_{S} \\
& =\left|\begin{array}{lc}
a\left\|\nabla u^{+}\right\|_{p}^{p}-\int_{\Omega} \lambda\left|u^{+}\right|^{p} d x & -b\left\|\nabla u^{+}\right\|_{p}^{p}\left\|\nabla u^{-}\right\|_{p}^{p} \\
a\left\|\nabla u^{-}\right\|_{p}^{p}-\int_{\Omega} \lambda\left|u^{-}\right|^{p} d x & \int_{\Omega}\left|u^{-}\right|^{2 p} d x-b\left\|\nabla u^{-}\right\|_{p}^{2 p}
\end{array}\right|
\end{aligned}
$$

$>0$.

Similarly, we have

$$
\begin{aligned}
& D_{T} \\
& =\left|\begin{array}{cc}
\int_{\Omega}\left|u^{+}\right|^{2 p} d x-b\left\|\nabla u^{+}\right\|_{p}^{2 p} & a\left\|\nabla u^{+}\right\|_{p}^{p}-\int_{\Omega} \lambda\left|u^{+}\right|^{p} d x \\
-b\left\|\nabla u^{+}\right\|_{p}^{p}\left\|\nabla u^{-}\right\|_{p}^{p} & a\left\|\nabla u^{-}\right\|_{p}^{p}-\int_{\Omega} \lambda\left|u^{-}\right|^{p} d x
\end{array}\right|
\end{aligned}
$$

$>0$.

From (21)-(23), we have $S=D_{S} / D>0, T=D_{T} / D>0$, and $(S, T)$ is the unique solution for system (20). Accordingly, $\left(s=S^{1 / p}, t=T^{1 / p}\right)$ is the unique positive solution for (18) and (19). Thus, (i) is proved.

(ii) Next, we give the proof of (ii).

From (6), we have

$$
\begin{aligned}
I_{b}\left(s u^{+}+t u^{-}\right)= & \frac{a s^{p}}{p}\left\|\nabla u^{+}\right\|_{p}^{p}+\frac{b s^{2 p}}{2 p}\left\|\nabla u^{+}\right\|_{p}^{2 p} \\
& -\frac{s^{p}}{p} \int_{\Omega} \lambda\left|u^{+}\right|^{p} d x \\
& -\frac{s^{2 p}}{2 p} \int_{\Omega}\left|u^{+}\right|^{2 p} d x+\frac{a t^{p}}{p}\left\|\nabla u^{-}\right\|_{p}^{p}
\end{aligned}
$$




$$
\begin{aligned}
& +\frac{b t^{2 p}}{2 p}\left\|\nabla u^{-}\right\|_{p}^{2 p}-\frac{t^{p}}{p} \int_{\Omega} \lambda\left|u^{-}\right|^{p} d x \\
& -\frac{t^{2 p}}{2 p} \int_{\Omega}\left|u^{-}\right|^{2 p} d x \\
& +\frac{b s^{p} t^{p}}{p}\left\|\nabla u^{+}\right\|_{p}^{p}\left\|\nabla u^{-}\right\|_{p}^{p} .
\end{aligned}
$$

By a simple computation, we have

$$
\begin{gathered}
\frac{\partial^{2} I_{b}}{\partial s^{2}}=(p-1) s^{p-2}\left\{a\left\|\nabla u^{+}\right\|_{p}^{p}-\lambda \int_{\Omega}\left|u^{+}\right|^{p} d x\right. \\
\left.+b t^{p}\left\|\nabla u^{+}\right\|_{p}^{p}\left\|\nabla u^{-}\right\|_{p}^{p}\right\}+(2 p-1) \\
\cdot s^{2 p-2}\left(b\left\|\nabla u^{+}\right\|_{p}^{2 p}-\int_{\Omega}\left|u^{+}\right|^{2 p} d x\right),
\end{gathered}
$$

and

$$
\begin{gathered}
\frac{\partial^{2} I_{b}}{\partial t^{2}}=(p-1) t^{p-2}\left\{a\left\|\nabla u^{-}\right\|_{p}^{p}-\lambda \int_{\Omega}\left|u^{-}\right|^{p} d x\right. \\
\left.+b s^{p}\left\|\nabla u^{+}\right\|_{p}^{p}\left\|\nabla u^{-}\right\|_{p}^{p}\right\}+(2 p-1) \\
\cdot t^{2 p-2}\left(b\left\|\nabla u^{-}\right\|_{p}^{2 p}-\int_{\Omega}\left|u^{-}\right|^{2 p} d x\right) .
\end{gathered}
$$

From $s_{u} u^{+}+t_{u} u^{-} \in \mathscr{M}_{b}$, we have

$$
\begin{aligned}
& \left.\frac{\partial^{2} I_{b}}{\partial s^{2}}\right|_{\left(s_{u}, t_{u}\right)}=p s_{u}^{2 p-2}\left(b\left\|\nabla u^{+}\right\|_{p}^{2 p}-\int_{\Omega}\left|u^{+}\right|^{2 p} d x\right)<0, \\
& \left.\frac{\partial^{2} I_{b}}{\partial t^{2}}\right|_{\left(s_{u}, t_{u}\right)}=p t_{u}^{2 p-2}\left(b\left\|\nabla u^{-}\right\|_{p}^{2 p}-\int_{\Omega}\left|u^{-}\right|^{2 p} d x\right)<0
\end{aligned}
$$

and

$$
\left.\frac{\partial^{2} I_{b}}{\partial t \partial s}\right|_{\left(s_{u}, t_{u}\right)}=p b s_{u}^{p-1} t_{u}^{p-1}\left\|\nabla u^{+}\right\|_{p}^{p}\left\|\nabla u^{-}\right\|_{p}^{p}
$$

We consider the Hessian matrix of $I_{b}\left(s u^{+}+t u^{-}\right)$; then from (17), we have

$$
\begin{gathered}
\left|H\left(s_{u}, t_{u}\right)\right|=\left|\begin{array}{ll}
\frac{\partial^{2} I_{b}}{\partial s^{2}} & \frac{\partial^{2} I_{b}}{\partial s \partial t} \\
\frac{\partial^{2} I_{b}}{\partial s \partial t} & \frac{\partial^{2} I_{b}}{\partial t^{2}}
\end{array}\right| \\
=p^{2} s_{u}^{2 p-2} t_{u}^{2 p-2}\left(\int_{\Omega}\left|u^{+}\right|^{2 p} d x-b\left\|\nabla u^{+}\right\|_{p}^{2 p}\right) \\
\cdot\left(\int_{\Omega}\left|u^{-}\right|^{2 p} d x-b\left\|\nabla u^{-}\right\|_{p}^{2 p}\right) \\
-p^{2} b^{2} s_{u}^{2 p-2} t_{u}^{2 p-2}\left\|\nabla u^{+}\right\|_{p}^{2 p}\left\|\nabla u^{-}\right\|_{p}^{2 p}>0 .
\end{gathered}
$$

The above deduction implies that $\left(s_{u}, t_{u}\right)$ is a maximal point of $I_{b}\left(s u^{+}+t u^{-}\right)$for $s, t \geq 0$. Since we cannot get the maximal point of $I_{b}$ on the boundary of $R^{+},\left(s_{u}, t_{u}\right)$ is the unique maximal point; that is, $I_{b}\left(s_{u} u^{+}+t_{u} u^{-}\right)=\max _{s, t \geq 0} I_{b}\left(s u^{+}+\right.$ $t u^{-}$).

Lemma 4. Assume that $\lambda<a \lambda_{1}$ and $u \in \mathscr{M}_{b}$, then (17) holds. Proof. For $u=u^{+}+u^{-} \in \mathscr{M}_{b}$, we have

$$
\begin{aligned}
\left\langle I_{b}^{\prime}(u), u^{+}\right\rangle= & a\left\|\nabla u^{+}\right\|_{p}^{p}+b\|\nabla u\|_{p}^{p}\left\|\nabla u^{+}\right\|_{p}^{p} \\
& -\int_{\Omega} \lambda\left|u^{+}\right|^{p} d x-\int_{\Omega}\left|u^{+}\right|^{2 p} d x=0, \\
\left\langle I_{b}^{\prime}(u), u^{-}\right\rangle= & a\left\|\nabla u^{-}\right\|_{p}^{p}+b\|\nabla u\|_{p}^{p}\left\|\nabla u^{-}\right\|_{p}^{p} \\
& -\int_{\Omega} \lambda\left|u^{-}\right|^{p} d x-\int_{\Omega}\left|u^{-}\right|^{2 p} d x=0 .
\end{aligned}
$$

Since $\lambda<a \lambda_{1}$, we have $a\left\|\nabla u^{ \pm}\right\|_{p}^{p}>\int_{\Omega} \lambda\left|u^{ \pm}\right|^{p} d x$. Thus, we have

$$
\begin{aligned}
& b\left\|\nabla u^{+}\right\|_{p}^{2 p}+b\left\|\nabla u^{+}\right\|_{p}^{p}\left\|\nabla u^{-}\right\|_{p}^{p}<\int_{\Omega}\left|u^{+}\right|^{2 p} d x \\
& b\left\|\nabla u^{-}\right\|_{p}^{2 p}+b\left\|\nabla u^{+}\right\|_{p}^{p}\left\|\nabla u^{-}\right\|_{p}^{p}<\int_{\Omega}\left|u^{-}\right|^{2 p} d x .
\end{aligned}
$$

Lemma 5. Assume that $b>0, \lambda<a \lambda_{1}, u \in W$ with $u^{ \pm} \neq 0$ and $\left\langle I_{b}^{\prime}(u), u^{ \pm}\right\rangle \leq 0$, there exists a unique pair $\left(s_{u}, t_{u}\right) \in(0,1] \times$ $(0,1]$ such that $s_{u} u^{+}+t_{u} u^{-} \in \mathscr{M}_{b}$.

Proof. If $u \in W$ with $u^{ \pm} \neq 0$ and $\left\langle I_{b}^{\prime}(u), u^{ \pm}\right\rangle \leq 0$, we have

$$
\begin{aligned}
& a\left\|\nabla u^{+}\right\|_{p}^{p}+b\|\nabla u\|_{p}^{p}\left\|\nabla u^{+}\right\|_{p}^{p} \\
& \quad \leq \int_{\Omega} \lambda\left|u^{+}\right|^{p} d x+\int_{\Omega}\left|u^{+}\right|^{2 p} d x \\
& a\left\|\nabla u^{-}\right\|_{p}^{p}+b\|\nabla u\|_{p}^{p}\left\|\nabla u^{-}\right\|_{p}^{p} \\
& \quad \leq \int_{\Omega} \lambda\left|u^{-}\right|^{p} d x+\int_{\Omega}\left|u^{-}\right|^{2 p} d x .
\end{aligned}
$$

Since $a\left\|\nabla u^{ \pm}\right\|_{p}^{p}>\lambda \int_{\Omega}\left|u^{ \pm}\right|^{p} d x$, then

$$
\begin{aligned}
& b\|\nabla u\|_{p}^{p}\left\|\nabla u^{+}\right\|_{p}^{p}<\int_{\Omega}\left|u^{+}\right|^{2 p} d x \\
& b\|\nabla u\|_{p}^{p}\left\|\nabla u^{-}\right\|_{p}^{p}<\int_{\Omega}\left|u^{-}\right|^{2 p} d x .
\end{aligned}
$$

From Lemma 3 , there is a unique pair $\left(s_{u}, t_{u}\right)$ of positive numbers such that $s_{u} u^{+}+t_{u} u^{-} \in \mathscr{M}_{b}$, which implies that $\left(s_{u}^{p}, t_{u}^{p}\right)$ is the solution of system (20). Then, we have

$$
\begin{aligned}
& D_{s_{u}^{p}}=\left(a\left\|\nabla u^{+}\right\|_{p}^{p}-\int_{\Omega} \lambda\left|u^{+}\right|^{p} d x\right) \\
& \cdot\left(\int_{\Omega}\left|u^{-}\right|^{2 p} d x-b\left\|\nabla u^{-}\right\|_{p}^{2 p}\right)+b\left\|\nabla u^{+}\right\|_{p}^{p}\left\|\nabla u^{-}\right\|_{p}^{p}
\end{aligned}
$$




$$
\begin{aligned}
& \cdot\left(a\left\|\nabla u^{-}\right\|_{p}^{p}-\int_{\Omega} \lambda\left|u^{-}\right|^{p} d x\right) \\
& \leq\left(\int_{\Omega}\left|u^{+}\right|^{2 p} d x-b\|\nabla u\|_{p}^{p}\left\|\nabla u^{+}\right\|_{p}^{p}\right) \\
& \cdot\left(\int_{\Omega}\left|u^{-}\right|^{2 p} d x-b\left\|\nabla u^{-}\right\|_{p}^{2 p}\right)+b\left\|\nabla u^{+}\right\|_{p}^{p}\left\|\nabla u^{-}\right\|_{p}^{p} \\
& \cdot\left(\int_{\Omega}\left|u^{-}\right|^{2 p} d x-b\|\nabla u\|_{p}^{p}\left\|\nabla u^{-}\right\|_{p}^{p}\right) \\
& =\left(\int_{\Omega}\left|u^{+}\right|^{2 p} d x-b\left\|\nabla u^{+}\right\|_{p}^{2 p}\right) \\
& \cdot\left(\int_{\Omega}\left|u^{-}\right|^{2 p} d x-b\left\|\nabla u^{-}\right\|_{p}^{2 p}\right)-b^{2}\left\|\nabla u^{+}\right\|_{p}^{2 p} \\
& \cdot\left\|\nabla u^{-}\right\|_{p}^{2 p}=D .
\end{aligned}
$$

Therefore, we have $s_{u}^{p}=D_{s_{u}^{p}} / D \leq 1$. Similarly, we have $t_{u}^{p}=$ $D_{t_{u}^{p}} / D \leq 1$. Thus, there exists a unique pair $\left(s_{u}, t_{u}\right) \in(0,1] \times$ $(0,1]$ such that $s_{u} u^{+}+t_{u} u^{-} \in M_{b}$.

Lemma 6. If $\lambda<a \lambda_{1}$ for any $u \in W$ with $b\|\nabla u\|_{p}^{2 p}<$ $\int_{\Omega}|u|^{2 p} d x$, there exists a unique $\overline{s_{u}}>0$ such that $\overline{s_{u}} u \in \mathcal{N}_{b}$. Moreover, $I_{b}\left(\overline{s_{u}} u\right)>I_{b}(s u)$ for all $s \geq 0$ and $s \neq \overline{s_{u}}$.

Proof. If $\lambda<a \lambda_{1}$ and $u \in W$ satisfies $b\|\nabla u\|_{p}^{2 p}<\int_{\Omega}|u|^{2 p} d x$, $s u \in \mathcal{N}_{b}$ implies that

$$
\begin{aligned}
\left\langle I_{b}^{\prime}(s u), s u\right\rangle= & a s^{p}\|\nabla u\|_{p}^{p}+b s^{2 p}\|\nabla u\|_{p}^{2 p} \\
& -s^{p} \int_{\Omega} \lambda|u|^{p} d x-s^{2 p} \int_{\Omega}|u|^{2 p} d x
\end{aligned}
$$

$=0$.

Thus, there exists a unique $\overline{s_{u}}=\left(\left(a\|\nabla u\|_{p}^{p}-\int_{\Omega} \lambda|u|^{p} d x\right) /\right.$ $\left.\left(\int_{\Omega}|u|^{2 p} d x-b\|\nabla u\|_{p}^{2 p}\right)\right)^{1 / p}>0$ satisfying (35). From (6), we have

$$
\begin{aligned}
I_{b}(s u)= & \frac{a s^{p}}{p}\|\nabla u\|_{p}^{p}+\frac{b s^{2 p}}{2 p}\|\nabla u\|_{p}^{2 p}-\frac{s^{p}}{p} \int_{\Omega} \lambda|u|^{p} d x \\
& -\frac{s^{2 p}}{2 p} \int_{\Omega}|u|^{2 p} d x .
\end{aligned}
$$

By a simple deduction, we have

$$
\begin{gathered}
\left.\frac{\partial^{2} I_{b}}{\partial s^{2}}\right|_{\overline{s_{u}}}=\left((p-1) s^{p-2}\left\{a\|\nabla u\|_{p}^{p}-\int_{\Omega} \lambda|u|^{p} d x\right\}\right. \\
\left.+(2 p-1) s^{2 p-2}\left(b\|\nabla u\|_{p}^{2 p}-\int_{\Omega}|u|^{2 p} d \mathrm{x}\right)\right)\left.\right|_{\overline{s_{u}}} \\
=-p{\overline{\bar{s}_{u}}}^{p-2}\left(a\|\nabla u\|_{p}^{p}-\int_{\Omega} \lambda|u|^{p} d x\right)<0 .
\end{gathered}
$$

Thus, $I_{b}(s u)$ attains its maximal point at $s=\overline{s_{u}}$. In other words, we have $I_{b}\left(\overline{s_{u}} u\right)>I_{b}(s u)$ for all $s \geq 0$ and $s \neq \overline{s_{u}}$.
Lemma 7. Assume $\lambda<a \lambda_{1}$; we have that

(i) if $0<b<1 / S^{2}, c_{b}>0$ is attained by some $v_{b} \in \mathcal{N}_{b}$ and $v_{b}$ is a constant sign critical point of $I_{b}$, where $S$ is given by (5);

(ii) if $0<b<1 / 2 S^{2}, m_{b}>0$ is attained by some $u_{b} \in M_{b}$ and $u_{b}$ is a sign-changing critical point of $I_{b}$.

Proof. (i) Firstly, we will show that for all $0<b<1 / S^{2}$, there exists $u \in W$ such that $b\|\nabla u\|_{p}^{2 p}<\int_{\Omega}|u|^{2 p} d x$, which implies $\mathcal{N}_{b} \neq \emptyset$. From (5), we know that there exists $e_{1} \in W$ such that $\left\|e_{1}\right\|_{2 p}=S^{-1 / p}\left\|e_{1}\right\|$. For $0<b<1 / S^{2}$, we have

$$
b\left\|\nabla e_{1}\right\|_{p}^{2 p}<\frac{1}{S^{2}}\left\|\nabla e_{1}\right\|_{p}^{2 p}=\int_{\Omega}\left|e_{1}\right|^{2 p} d x
$$

Thus, we have $\mathcal{N}_{b} \neq \emptyset$.

For each $u \in \mathcal{N}_{b}$, it follows from $\lambda<a \lambda_{1}$ and (5) that

$$
\begin{aligned}
a\|\nabla u\|_{p}^{p}+b\|\nabla u\|_{p}^{2 p} & =\lambda \int_{\Omega}|u|^{p} d x+\int_{\Omega}|u|^{2 p} d x \\
& \leq \frac{\lambda}{\lambda_{1}}\|\nabla u\|_{p}^{p}+\frac{1}{S^{2}}\|\nabla u\|_{p}^{2 p} .
\end{aligned}
$$

Then, $\|\nabla u\|_{p}^{p} \geq\left(a-\lambda / \lambda_{1}\right) /\left(1 / S^{2}-b\right)$. Thus, we have

$$
\begin{aligned}
I_{b}(u) & =I_{b}(u)-\frac{1}{2 p}\left\langle I_{b}^{\prime}(u), u\right\rangle \\
& =\frac{1}{2 p}\left(a\|\nabla u\|_{p}^{p}-\int_{\Omega} \lambda|u|^{p} d x\right) \\
& \geq \frac{1}{2 p}\left(a-\frac{\lambda}{\lambda_{1}}\right)\|\nabla u\|_{p}^{p} \geq \frac{\left(a-\lambda / \lambda_{1}\right)^{2} S^{2}}{2 p\left(1-b S^{2}\right)},
\end{aligned}
$$

that is, $c_{b}=\inf _{u \in \mathcal{N}_{b}} I_{b}(u) \geq\left(a-\lambda / \lambda_{1}\right)^{2} S^{2} / 2 p\left(1-b S^{2}\right)>0$ and $I_{b}$ is coercive and bounded below on $\mathcal{N}_{b}$ for $0<b<1 / S^{2}$ and $\lambda<a \lambda_{1}$.

Let $\left\{v_{n}\right\} \subset \mathcal{N}_{b}$ be a minimizing sequence for $I_{b}$. From $I_{b}\left(v_{n}\right)=I_{b}\left(\left|v_{n}\right|\right)$ and $\left|v_{n}\right| \in \mathcal{N}_{b}$, we assume that $v_{n}(x) \geq 0$ in $\Omega$ for all $n \in \mathbb{N}$. Since $I_{b}$ is coercive and bounded below on $\mathcal{N}_{b}$, the sequence $\left\{v_{n}\right\}$ is bounded in $W$, so that, up to subsequences, $v_{n} \rightarrow v_{b}$ in $W$ and $v_{b} \geq 0$. Next, we will prove that $v_{n} \longrightarrow v_{b}$ strongly in $W$. We suppose by contradiction that $\left\|v_{b}\right\|<\liminf _{n \rightarrow \infty}\left\|v_{n}\right\|$. Therefore, we have

$$
a\left\|\nabla v_{b}\right\|_{p}^{p}+b\left\|\nabla v_{b}\right\|_{p}^{2 p}<\lambda \int_{\Omega}\left|v_{b}\right|^{p} d x+\int_{\Omega}\left|v_{b}\right|^{2 p} d x
$$

If $v_{b}=0$, the above inequality makes a contradiction. Thus, we have $v_{b} \neq 0$ in $\Omega$. From the fact that $a\left\|\nabla v_{b}\right\|_{p}^{p}>$ $\lambda \int_{\Omega}\left|v_{b}\right|^{p} d x$, we have $b\left\|\nabla v_{b}\right\|_{p}^{2 p}<\int_{\Omega}\left|v_{b}\right|^{2 p} d x$. By Lemma 6, 
there exists a unique $s_{v}>0$ such that $s_{v} v_{b} \in \mathcal{N}_{b}$ and $I_{b}\left(s_{v} v_{n}\right) \leq$ $I_{b}\left(v_{n}\right)$ for all $v_{n} \in \mathcal{N}_{b}$. Thus, we have

$$
\begin{aligned}
c_{b} & \leq I_{b}\left(s_{v} v_{b}\right)=\frac{a}{p}\left\|\nabla\left(s_{v} v_{b}\right)\right\|_{p}^{p}+\frac{b}{2 p}\left\|\nabla\left(s_{v} v_{b}\right)\right\|_{p}^{2 p}-\frac{\lambda}{p} \\
\cdot & \int_{\Omega}\left|s_{v} v_{b}\right|^{p} d x-\frac{1}{2 p} \int_{\Omega}\left|s_{v} v_{b}\right|^{2 p} d x \\
& <\liminf _{n \rightarrow \infty}\left(\frac{a}{p}\left\|\nabla\left(s_{v} v_{n}\right)\right\|_{p}^{p}+\frac{b}{2 p}\left\|\nabla\left(s_{v} v_{n}\right)\right\|_{p}^{2 p}\right. \\
& \left.-\frac{\lambda}{p} \int_{\Omega}\left|s_{v} v_{n}\right|^{p} d x-\frac{1}{2 p} \int_{\Omega}\left|s_{v} v_{n}\right|^{2 p} d x\right) \\
& =\liminf _{n \longrightarrow \infty} I_{b}\left(s_{v} v_{n}\right) \leq \liminf _{n \longrightarrow \infty} I_{b}\left(v_{n}\right)=c_{b},
\end{aligned}
$$

which leads to a contradiction. Therefore, we have $\left\|v_{b}\right\|=$ $\liminf _{n \rightarrow \infty}\left\|v_{n}\right\|, v_{n} \longrightarrow v_{b}$ strongly in $W$ and $I_{b}\left(v_{b}\right)=$ $c_{b}$. Then, by a standard argument, which is similar to the discussion in [34], we can deduce that $v_{b}$ is a constant sign critical point of $I_{b}$.

(ii) From a similar deduction as (i), we know that for $0<$ $b<1 / 2 S^{2}$, there exists $u_{1} \in W$ such that

$$
b\left\|\nabla u_{1}\right\|_{p}^{2 p}<\frac{1}{2 S^{2}}\left\|\nabla u_{1}\right\|_{p}^{2 p}=\frac{1}{2} \int_{\Omega}\left|u_{1}\right|^{2 p} d x .
$$

Obviously, if $u \in W$ such that $u_{1}$ satisfies (43), then $\left|u_{1}\right| \in W$ also satisfies (43). Therefore, we assume that $u_{1}(x) \geq 0$ a.e. in $W$. We let suppu $u_{1} \subset B_{\rho}\left(x_{0}\right)$ and define $u_{2}(x)=-u_{1}(-x)$ for all $x \in B_{\rho}\left(-x_{0}\right)$, where $B_{\rho}\left(x_{0}\right)=\left\{x \in \Omega:\left|x-x_{0}\right|<\rho\right\}$ and $\rho>0$. Then, from (43), we have

$$
\frac{\int_{\Omega}\left|u_{1}\right|^{2 p} d x}{\left\|\nabla u_{1}\right\|_{p}^{2 p}}=\frac{\int_{\Omega}\left|u_{2}\right|^{2 p} d x}{\left\|\nabla u_{2}\right\|_{p}^{2 p}}>2 b .
$$

Let $u=u_{1}+u_{2}$; we can obtain that $u \in W$ and $u^{+}=u_{1}, u^{-}=$ $u_{2}$ and

$$
\begin{aligned}
b \| & \nabla u^{+}\left\|_{p}^{2 p}+b\right\| \nabla u^{+}\left\|_{p}^{p}\right\| \nabla u^{-} \|_{p}^{p} \\
& <\frac{1}{2} \int_{\Omega}\left|u_{1}\right|^{2 p} d x+\frac{1}{2}\left\|u_{1}\right\|_{2 p}^{p}\left\|u_{2}\right\|_{2 p}^{p} \\
& =\int_{\Omega}\left|u_{1}\right|^{2 p} d x,
\end{aligned}
$$

that is,

$$
b\left\|\nabla u^{+}\right\|_{p}^{2 p}+b\left\|\nabla u^{+}\right\|_{p}^{p}\left\|\nabla u^{-}\right\|_{p}^{p}<\int_{\Omega}\left|u^{+}\right|^{2 p} d x .
$$

Similarly, we also have

$$
b\left\|\nabla u^{-}\right\|_{p}^{2 p}+b\left\|\nabla u^{+}\right\|_{p}^{p}\left\|\nabla u^{-}\right\|_{p}^{p}<\int_{\Omega}\left|u^{-}\right|^{2 p} d x .
$$

By Lemma 3, we know that $\mathscr{M}_{b} \neq \emptyset$ for $0<b<1 / 2 S^{2}$.

Assume that $\left\{u_{n}\right\} \subset \mathscr{M}_{b}$ is a minimizing sequence for $I_{b}$, such that $I_{b}\left(u_{n}\right) \longrightarrow m_{b}$. Since $I_{b}$ is coercive on $\mathcal{N}_{b}$, the sequence $\left\{u_{n}\right\}$ is bounded in $W$; going if necessary to a subsequence, still denoted by $\left\{u_{n}\right\}$, we can assume that there exists a $u_{b} \in W$ such that for $n$ sufficiently large,

$$
\begin{aligned}
u_{n}^{ \pm} & \rightarrow u_{b}^{ \pm} \quad \text { weakly in } W, \\
u_{n}(x) & \longrightarrow u_{b}(x) \quad \text { almost everywhere on } \Omega, \\
u_{n}^{ \pm} & \longrightarrow u_{\mathrm{b}}^{ \pm} \quad \text { strongly in } L^{s}(\Omega) \text { for } p \leq s<p^{*} .
\end{aligned}
$$

From $\left\{u_{n}\right\} \subset \mathscr{M}_{b}$, we have $\left\langle I_{b}^{\prime}\left(u_{n}\right), u_{n}^{ \pm}\right\rangle=0$; that is,

$$
\begin{aligned}
& a\left\|\nabla u_{n}^{ \pm}\right\|_{p}^{p}+b\left\|\nabla u_{n}\right\|_{p}^{p}\left\|\nabla u_{n}^{ \pm}\right\|_{p}^{p} \\
& \quad=\lambda \int_{\Omega}\left|u_{n}^{ \pm}\right|^{p} d x+\int_{\Omega}\left|u_{n}^{ \pm}\right|^{2 p} d x .
\end{aligned}
$$

Therefore,

$$
a\left\|\nabla u_{n}^{ \pm}\right\|_{p}^{p} \leq \lambda \int_{\Omega}\left|u_{n}^{ \pm}\right|^{p} d x+\int_{\Omega}\left|u_{n}^{ \pm}\right|^{2 p} d x .
$$

In the same way, we have $\left(a-\lambda / \lambda_{1}\right)\left\|\nabla u_{n}^{ \pm}\right\|_{p}^{p} \leq\left(1 / S^{2}\right)\left\|\nabla u_{n}^{ \pm}\right\|_{p}^{2 p}$ and $\left\|\nabla u_{n}^{ \pm}\right\|_{p}^{p} \geq S^{2}\left(a-\lambda / \lambda_{1}\right)>0$. Passing to the limit, we have

$$
\begin{aligned}
0 & <S^{2}\left(a-\frac{\lambda}{\lambda_{1}}\right)^{2} \leq \liminf _{n \rightarrow \infty}\left(a-\frac{\lambda}{\lambda_{1}}\right)\left\|\nabla u_{n}^{ \pm}\right\|_{p}^{p} \\
& \leq \int_{\Omega}\left|u_{b}^{ \pm}\right|^{2 p} d x,
\end{aligned}
$$

which implies that $u_{b}^{ \pm} \neq 0$ and

$$
\begin{aligned}
& a\left\|\nabla u_{b}^{+}\right\|_{p}^{p}+b\left\|\nabla u_{b}\right\|_{p}^{p}\left\|\nabla u_{b}^{+}\right\|_{p}^{p} \\
& \leq \lambda \int_{\Omega}\left|u_{b}^{+}\right|^{p} d x+\int_{\Omega}\left|u_{b}^{+}\right|^{2 p} d x, \\
& a\left\|\nabla u_{b}^{-}\right\|_{p}^{p}+b\left\|\nabla u_{b}\right\|_{p}^{p}\left\|\nabla u_{b}^{-}\right\|_{p}^{p} \\
& \leq \lambda \int_{\Omega}\left|u_{b}^{-}\right|^{p} d x+\int_{\Omega}\left|u_{b}^{-}\right|^{2 p} d x .
\end{aligned}
$$

From $a\left\|\nabla u_{b}^{ \pm}\right\|_{p}^{p}>\lambda \int_{\Omega}\left|u_{b}^{ \pm}\right|^{p} d x$, Lemmas 3 and 5 , there exists a unique pair $\left(s_{u}, t_{u}\right) \in(0,1] \times(0,1]$ such that

$$
s_{u} u_{b}^{+}+t_{u} u_{b}^{-} \in \mathscr{M}_{b} .
$$

From the definition of $m_{b}$, we have

$$
\begin{aligned}
m_{b} & \leq I_{b}\left(s_{u} u_{b}^{+}+t_{u} u_{b}^{-}\right)=I_{b}\left(s_{u} u_{b}^{+}+t_{u} u_{b}^{-}\right) \\
& -\frac{1}{2 p}\left\langle I_{b}^{\prime}\left(s_{u} u_{b}^{+}+t_{u} u_{b}^{-}\right), s_{u} u_{b}^{+}+t_{u} u_{b}^{-}\right\rangle \\
& =\frac{1}{2 p}\left(a\left\|\nabla\left(s_{u} u_{b}^{+}+t_{u} u_{b}^{-}\right)\right\|_{p}^{p}\right. \\
& \left.-\lambda \int_{\Omega}\left|s_{u} u_{b}^{+}+t_{u} u_{b}^{-}\right|^{p} d x\right)
\end{aligned}
$$




$$
\begin{aligned}
& =\frac{1}{2 p}\left\{s_{u}^{p}\left(a\left\|\nabla u_{b}^{+}\right\|_{p}^{p}-\lambda \int_{\Omega}\left|u_{b}^{+}\right|^{p} d x\right)\right. \\
& \left.+t_{u}^{p}\left(a\left\|\nabla u_{b}^{-}\right\|_{p}^{p}-\lambda \int_{\Omega}\left|u_{b}^{-}\right|^{p} d x\right)\right\} \\
& \leq \frac{1}{2 p}\left\{\left(a\left\|\nabla u_{b}^{+}\right\|_{p}^{p}-\lambda \int_{\Omega}\left|u_{b}^{+}\right|^{p} d x\right)\right. \\
& \left.+\left(a\left\|\nabla u_{b}^{-}\right\|_{p}^{p}-\lambda \int_{\Omega}\left|u_{b}^{-}\right|^{p} d x\right)\right\}=\frac{1}{2 p}\left(a\left\|\nabla u_{b}\right\|_{p}^{p}\right. \\
& \left.-\lambda \int_{\Omega}\left|u_{b}\right|^{p} d x\right) \\
& \leq \liminf _{n \longrightarrow \infty}\left\{I_{b}\left(u_{n}\right)-\frac{1}{2 p}\left\langle I_{b}^{\prime}\left(u_{n}\right), u_{n}\right\rangle\right\}=m_{b} .
\end{aligned}
$$

Thus, $s_{u}=t_{u}=1, u_{b} \in \mathscr{M}_{b}$, and $I_{b}\left(u_{b}\right)=m_{b}, u_{b}$ is the required minimizer.

Next, we will prove that $u_{b}$ is indeed a sign-changing solution; that is, $I_{b}^{\prime}\left(u_{b}\right)=0$. We mainly use the quantitative deformation lemma [35] to prove the results.

If $I_{b}^{\prime}\left(u_{b}\right) \neq 0$, there exists $\delta>0$ and $\alpha>0$, such that

$$
\begin{aligned}
u & \in W, \\
\left\|I_{b}^{\prime}(u)\right\| & \geq \alpha, \\
\forall\left\|u-u_{b}\right\| & \leq 3 \delta .
\end{aligned}
$$

Let $D=(1 / 2,3 / 2) \times(1 / 2,3 / 2), \psi(s, t)=s u_{b}^{+}+t u_{b}^{-}$, and $(s, t) \epsilon$ $D$. It follows from Lemma 3 that

$$
\overline{m_{b}}=\max _{\partial D} I_{b} \circ \psi<m_{b} \text {. }
$$

Let $\varepsilon=\min \left\{\left(m_{b}-\overline{m_{b}}\right) / 3, \alpha \delta / 8\right\}$ and $S_{\delta}=\left\{u \in W:\left\|u-u_{b}\right\| \leq\right.$ $\delta\}$; there exists a deformation $\eta \in C([0,1] \times W, W)$ such that

(i) $\eta(1, u)=u$ if $u \notin I_{b}^{-1}\left(\left[m_{b}-2 \varepsilon, m_{b}+2 \varepsilon\right]\right) \cap S_{2 \delta}$;

(ii) $\eta\left(1, I_{b}^{m_{b}+\varepsilon} \cap S_{\delta}\right) \subset I_{b}^{m_{b}-\varepsilon}$;

(iii) $I_{b}(\eta(1, u)) \leq I_{b}(u), \forall u \in W$.

From (56), Lemma 3 and (ii), we can easily get

$$
\max _{(s, t) \in \bar{D}} I_{b}(\eta(1, \psi(s, t)))<m_{b}
$$

We prove that $\eta(1, \psi(D)) \cap \mathscr{M}_{b} \neq \emptyset$, which contradicts the definition of $m_{b}$. We define $g(s, t)=\eta(1, \psi(s, t))$ and

$$
\begin{aligned}
& \Phi_{0}(s, t)=\left(I_{b}^{\prime}(\psi(s, t)) u_{b}^{+}, I_{b}^{\prime}(\psi(s, t)) u_{b}^{-}\right) \\
& \quad=\left(I_{b}^{\prime}\left(s u_{b}^{+}+t u_{b}^{-}\right) u_{b}^{+}, I_{b}^{\prime}\left(s u_{b}^{+}+t u_{b}^{-}\right) u_{b}^{-}\right), \\
& \Phi_{1}(s, t) \\
& =\left(\frac{1}{s} I_{b}^{\prime}(g(s, t)) g^{+}(s, t), \frac{1}{t} I_{b}^{\prime}(g(s, \mathrm{t})) g^{-}(s, t)\right) .
\end{aligned}
$$

Lemma 3 and the degree theory yield $\operatorname{deg}\left(\Phi_{0}, D, 0\right)=1$. From (56), we know that $g=\psi$ on $\partial D$. Consequently, we have $\operatorname{deg}\left(\Phi_{0}, D, 0\right)=\operatorname{deg}\left(\Phi_{1}, D, 0\right)=1$. Therefore, $\Phi_{1}\left(s_{0}, t_{0}\right)=0$ for some $\left(s_{0}, t_{0}\right) \in D$; that is, $\eta\left(1, \psi\left(s_{0}, t_{0}\right)\right)=g\left(s_{0}, t_{0}\right) \in$ $\mathscr{M}_{b}$, which is a contradiction. From this point, $u_{b}$ is a signchanging critical point of $I_{b}$ and $I_{b}^{\prime}\left(u_{b}\right)=0$.

\section{The Existence of the Sign-Changing Solutions}

In this part, we are devoted to proving Theorem 1.

Proof of Theorem 1. In view of Lemma 7, we know that for $0<b<1 / 2 S^{2}$ and $\lambda<a \lambda_{1}$, there exists a $u_{b} \in \mathscr{M}_{b}$ such that $m_{b}=I_{b}\left(u_{b}\right)$ and $I_{b}^{\prime}\left(u_{b}\right)=0$; that is, $u_{b}$ is a ground state sign-changing solution for problem (1). Then by Lemma 4 , we have that

$$
\begin{aligned}
& b\left\|\nabla u_{b}^{+}\right\|_{p}^{2 p}+b\left\|\nabla u_{b}^{+}\right\|_{p}^{p}\left\|\nabla u_{b}^{-}\right\|_{p}^{p}<\int_{\Omega}\left|u_{b}^{+}\right|^{2 p} d x \\
& b\left\|\nabla u_{b}^{-}\right\|_{p}^{2 p}+b\left\|\nabla u_{b}^{+}\right\|_{p}^{p}\left\|\nabla u_{b}^{-}\right\|_{p}^{p}<\int_{\Omega}\left|u_{b}^{-}\right|^{2 p} d x,
\end{aligned}
$$

which implies

$$
\begin{aligned}
& b\left\|\nabla u_{b}^{+}\right\|_{p}^{2 p}<\int_{\Omega}\left|u_{b}^{+}\right|^{2 p} d x \\
& b\left\|\nabla u_{b}^{-}\right\|_{p}^{2 p}<\int_{\Omega}\left|u_{b}^{-}\right|^{2 p} d x .
\end{aligned}
$$

Then from Lemma 6 , there exist $s_{1}, t_{1}>0$ such that $s_{1} u_{b}^{+}, t_{1} u_{b}^{-} \in \mathcal{N}_{b}$. Therefore, we have

$$
\begin{aligned}
m_{b} & =I_{b}\left(u_{b}\right) \geq I_{b}\left(s_{1} u_{b}^{+}+t_{1} u_{b}^{-}\right) \\
& =I_{b}\left(s_{1} u_{b}^{+}\right)+I_{b}\left(t_{1} u_{b}^{-}\right)+\frac{b s_{1}^{p} t_{1}^{p}}{p}\left\|\nabla u_{b}^{+}\right\|_{p}^{p}\left\|\nabla u_{b}^{-}\right\|_{p}^{p} \\
& >I_{b}\left(s_{1} u_{b}^{+}\right)+I_{b}\left(t_{1} u_{b}^{-}\right) \geq 2 c_{b} .
\end{aligned}
$$

Therefore, the energy doubling property is proved.

Next, we prove that $u_{b}$ changes sign only once; that is, $u_{b}$ has exactly two nodal domains. We assume by contradiction that $u_{b}=u_{1}+u_{2}+u_{3}$ with $u_{i} \neq 0, u_{1} \geq 0, u_{2} \leq 0, u_{3} \geq 0$ and $\operatorname{supp}\left(u_{i}\right) \cap \operatorname{supp}\left(u_{j}\right)=\emptyset$ for $i \neq j,(i, j=1,2,3)$.

Since $I_{b}^{\prime}\left(u_{b}\right)=0$, we can get

$$
\begin{aligned}
\left\langle I_{b}^{\prime}\left(u_{1}+u_{2}\right), u_{1}\right\rangle= & \left\langle I_{b}^{\prime}\left(u_{b}\right), u_{1}\right\rangle \\
& -b\left\|\nabla u_{1}\right\|_{p}^{p}\left\|\nabla u_{3}\right\|_{p}^{p}<0, \\
\left\langle I_{b}^{\prime}\left(u_{1}+u_{2}\right), u_{2}\right\rangle= & \left\langle I_{b}^{\prime}\left(u_{b}\right), u_{2}\right\rangle \\
& -b\left\|\nabla u_{2}\right\|_{p}^{p}\left\|\nabla u_{3}\right\|_{p}^{p}<0 .
\end{aligned}
$$

Then, by Lemma 5 , there exists a pair $\left(s^{\prime}, t^{\prime}\right) \in(0,1] \times(0,1]$ such that $s^{\prime} u_{1}+t^{\prime} u_{2} \in M_{b}$ and $I_{b}\left(s^{\prime} u_{1}+t^{\prime} u_{2}\right) \geq m_{b}$.

From $\lambda<a \lambda_{1},\left\langle I_{b}^{\prime}\left(u_{b}\right), u_{b}\right\rangle=0$, and $\left\langle I_{b}^{\prime}\left(s^{\prime} u_{1}+t^{\prime} u_{2}\right), s^{\prime} u_{1}+\right.$ $\left.t^{\prime} u_{2}\right\rangle=0$, we have

$$
\begin{aligned}
m_{b} & =I_{b}\left(u_{b}\right)-\frac{1}{2 p}\left\langle I_{b}^{\prime}\left(u_{b}\right), u_{b}\right\rangle=\frac{1}{2 p}\left(a\left\|\nabla u_{b}\right\|_{p}^{p}\right. \\
& \left.-\lambda \int_{\Omega}\left|u_{b}\right|^{p} d x\right) \\
& =\frac{1}{2 p}\left\{\left(a\left\|\nabla u_{1}\right\|_{p}^{p}-\lambda \int_{\Omega}\left|u_{1}\right|^{p} d x\right)\right.
\end{aligned}
$$




$$
\begin{aligned}
& +\left(a\left\|\nabla u_{2}\right\|_{p}^{p}-\lambda \int_{\Omega}\left|u_{2}\right|^{p} d x\right) \\
& \left.+\left(a\left\|\nabla u_{3}\right\|_{p}^{p}-\lambda \int_{\Omega}\left|u_{3}\right|^{p} d x\right)\right\} \\
& >\frac{1}{2 p}\left\{\left(a\left\|\nabla u_{1}\right\|_{p}^{p}-\lambda \int_{\Omega}\left|u_{1}\right|^{p} d x\right)\right. \\
& \left.+\left(a\left\|\nabla u_{2}\right\|_{p}^{p}-\lambda \int_{\Omega}\left|u_{2}\right|^{p} d x\right)\right\} \\
& \geq \frac{1}{2 p}\left\{\left(s^{\prime}\right)^{p}\left(a\left\|\nabla u_{1}\right\|_{p}^{p}-\lambda \int_{\Omega}\left|u_{1}\right|^{p} d x\right)\right. \\
& \left.+\left(t^{\prime}\right)^{p}\left(a\left\|\nabla u_{2}\right\|_{p}^{p}-\lambda \int_{\Omega}\left|u_{2}\right|^{p} d x\right)\right\}=I_{b}\left(s^{\prime} u_{1}\right. \\
& \left.+t^{\prime} u_{2}\right)-\frac{1}{2 p}\left\langle I_{b}^{\prime}\left(s^{\prime} u_{1}+t^{\prime} u_{2}\right), s^{\prime} u_{1}+t^{\prime} u_{2}\right\rangle \\
& =I_{b}\left(s^{\prime} u_{1}+t^{\prime} u_{2}\right) \geq m_{b},
\end{aligned}
$$

which leads to a contradiction; thus, $u_{b}$ has exactly two nodal domains.

\section{The Convergence Property of $u_{b}$ as $b \searrow 0$}

In this part, we regard $b>0\left(b \in\left(0,1 / 2 S^{2}\right)\right)$ as a small parameter in (1) and discuss the convergence property of the least energy sign-changing solution $u_{b}$, where $u_{b} \in \mathscr{M}_{b}$ and $u_{b}$ changes sign only once.

Proof of Theorem 2. We choose a nonzero function $w_{0} \in$ $C_{0}^{\infty}(\Omega)$ and $\gamma>0$ such that $w_{0}^{ \pm} \neq 0$ and

$$
\begin{aligned}
& a\left\|\nabla w_{0}^{+}\right\|_{p}^{p}+\gamma\left\|\nabla w_{0}\right\|_{p}^{p}\left\|\nabla w_{0}^{+}\right\|_{p}^{p} \\
& \leq \lambda \int_{\Omega}\left|w_{0}^{+}\right|^{p} d x+\int_{\Omega}\left|w_{0}^{+}\right|^{2 p} d x \\
& a\left\|\nabla w_{0}^{-}\right\|_{p}^{p}+\gamma\left\|\nabla w_{0}\right\|_{p}^{p}\left\|\nabla w_{0}^{-}\right\|_{p}^{p} \\
& \quad \leq \lambda \int_{\Omega}\left|w_{0}^{-}\right|^{p} d x+\int_{\Omega}\left|w_{0}^{-}\right|^{2 p} d x .
\end{aligned}
$$

Thus, for any $b \in[0, \gamma]$, we have $\left\langle I_{b}^{\prime}\left(w_{0}\right), w_{0}^{ \pm}\right\rangle \leq 0$. It follows from Lemma 5 that there is a unique pair $\left(s_{b}, t_{b}\right) \in(0,1] \times$ $(0,1]$ such that $s_{b} w_{0}^{+}+t_{b} w_{0}^{-} \in \mathscr{M}_{b}$. Thus, we have

$$
\begin{aligned}
& I_{b}\left(s_{b} w_{0}^{+}+t_{b} w_{0}^{-}\right)=I_{b}\left(s_{b} w_{0}^{+}+t_{b} w_{0}^{-}\right) \\
& \quad-\frac{1}{2 p}\left\langle I_{b}^{\prime}\left(s_{b} w_{0}^{+}+t_{b} w_{0}^{-}\right), s_{b} w_{0}^{+}+t_{b} w_{0}^{-}\right\rangle \\
& \quad=\frac{1}{2 p}\left(a\left\|\nabla\left(s_{b} w_{0}^{+}+t_{b} w_{0}^{-}\right)\right\|_{p}^{p}\right. \\
& \left.\quad-\lambda \int_{\Omega}\left|s_{b} w_{0}^{+}+t_{b} w_{0}^{-}\right|^{p} d x\right) \\
& \quad<\frac{a}{2 p}\left\|\nabla\left(s_{b} w_{0}^{+}+t_{b} w_{0}^{-}\right)\right\|_{p}^{p} \leq \frac{a}{2 p}\left\|w_{0}\right\|^{p}=\Theta .
\end{aligned}
$$

For any sequence $\left\{b_{n}\right\}$ with $b_{n} \searrow 0$ as $n \longrightarrow \infty$, there exists $u_{b_{n}} \in \mathscr{M}_{b}$ such that $u_{b_{n}}$ is a ground state sign-changing critical point of $I_{b_{n}}(u)$ and

$$
\begin{aligned}
\Theta+1 & \geq I_{b_{n}}\left(u_{b_{n}}\right)-\frac{1}{2 p}\left\langle I_{b_{n}}^{\prime}\left(u_{b_{n}}\right), u_{b_{n}}\right\rangle \\
& =\frac{1}{2 p}\left(a\left\|\nabla u_{b_{n}}\right\|_{p}^{p}-\lambda \int_{\Omega}\left|u_{b_{n}}\right|^{p} d x\right) \\
& \geq \frac{1}{2 p}\left(a-\frac{\lambda}{\lambda_{1}}\right)\left\|\nabla u_{b_{n}}\right\|_{p}^{p} .
\end{aligned}
$$

The above inequality shows that $u_{b_{n}}$ is bounded in $W$; then there exists a subsequence of $\left\{b_{n}\right\}$, still denoted by $\left\{b_{n}\right\}$, such that $u_{b_{n}} \rightarrow u_{0}$ weakly in $W$. By the compactness of the embedding $W \hookrightarrow L^{s}(\Omega)$ for $p \leq s<p^{*}$, using a standard argument, we have that $u_{b_{n}}^{ \pm} \longrightarrow u_{0}^{ \pm}$in $W$ and $u_{0}^{ \pm} \neq 0$. Moreover, we have that for all $u \in W$,

$$
\begin{aligned}
0= & \lim _{n \longrightarrow \infty}\left\langle I_{b_{n}}^{\prime}\left(u_{b_{n}}\right), u\right\rangle \\
& =\lim _{n \rightarrow \infty}\left\{\int_{\Omega} a\left|\nabla u_{b_{n}}\right|^{p-2} \nabla u_{b_{n}} \nabla u d x\right. \\
& +b_{n} \int_{\Omega}\left|\nabla u_{b_{n}}\right|^{p} d x \int_{\Omega}\left|\nabla u_{b_{n}}\right|^{p-2} \nabla u_{b_{n}} \nabla u d x \\
& \left.-\lambda \int_{\Omega}\left|u_{b_{n}}\right|^{p-2} u_{b_{n}} u d x-\int_{\Omega}\left|u_{b_{n}}\right|^{2 p-2} u_{b_{n}} u d x\right\} \\
& =\int_{\Omega} a\left|\nabla u_{0}\right|^{p-2} \nabla u_{0} \nabla u d x-\lambda \int_{\Omega}\left|u_{0}\right|^{p-2} u_{0} u d x \\
& -\int_{\Omega}\left|u_{0}\right|^{2 p-2} u_{0} u d x=\left\langle I_{0}^{\prime}\left(u_{0}\right), u\right\rangle,
\end{aligned}
$$

which implies that

$$
I_{0}^{\prime}\left(u_{0}\right)=0, \quad u_{0} \in \mathscr{M}_{0}, I_{0}\left(u_{0}\right) \geq m_{0}
$$

Secondly, in the Proof of Theorem $1, b=0$ is allowed; then, there exists a $\nu_{0} \in \mathscr{M}_{0}$ such that

$$
I_{0}\left(\nu_{0}\right)=m_{0}=\inf _{u \in \mathscr{M}_{0}} I_{0}(u)
$$

and $v_{0}$ is a sign-changing solution for (11) which changes sign only once. Similarly, we can pick up $\varepsilon>0$ which is independent of $b_{n}$ such that

$$
\begin{aligned}
& \varepsilon\left\|\nabla v_{0}^{+}\right\|_{p}^{2 p}+\varepsilon\left\|\nabla v_{0}^{+}\right\|_{p}^{p}\left\|\nabla v_{0}^{-}\right\|_{p}^{p}<\int_{\Omega}\left|v_{0}^{+}\right|^{2 p} d x \\
& \varepsilon\left\|\nabla v_{0}^{-}\right\|_{p}^{2 p}+\varepsilon\left\|\nabla v_{0}^{+}\right\|_{p}^{p}\left\|\nabla v_{0}^{-}\right\|_{p}^{p}<\int_{\Omega}\left|v_{0}^{-}\right|^{2 p} d x .
\end{aligned}
$$

According to Lemma 3, there exists a unique pair $\left(s_{0}, t_{0}\right)$ of positive numbers such that $s_{0} \nu_{0}^{+}+t_{0} \nu_{0}^{-} \in \mathscr{M}_{\varepsilon}$. 
Let $b_{n} \in[0, \varepsilon]$; we know that

$$
\begin{aligned}
\left\langle I_{b_{n}}^{\prime}\right. & \left.\left(s_{0} v_{0}^{+}+t_{0} v_{0}^{-}\right), s_{0} v_{0}^{+}\right\rangle \\
= & a\left\|\nabla\left(s_{0} v_{0}^{+}\right)\right\|_{p}^{p}+b_{n}\left\|\nabla\left(s_{0} v_{0}^{+}\right)\right\|_{p}^{2 p} \\
& +b_{n}\left\|\nabla\left(s_{0} v_{0}^{+}\right)\right\|_{p}^{p}\left\|\nabla\left(t_{0} v_{0}^{-}\right)\right\|_{p}^{p}-\lambda \int_{\Omega}\left|s_{0} v_{0}^{+}\right|^{p} d x \\
& \quad-\int_{\Omega}\left|s_{0} v_{0}^{+}\right|^{2 p} d x \\
\leq & a\left\|\nabla\left(s_{0} v_{0}^{+}\right)\right\|_{p}^{p}+\varepsilon\left\|\nabla\left(s_{0} v_{0}^{+}\right)\right\|_{p}^{2 p} \\
& +\varepsilon\left\|\nabla\left(s_{0} v_{0}^{+}\right)\right\|_{p}^{p}\left\|\nabla\left(t_{0} v_{0}^{-}\right)\right\|_{p}^{p}-\lambda \int_{\Omega}\left|s_{0} v_{0}^{+}\right|^{p} d x \\
& \quad-\int_{\Omega}\left|s_{0} v_{0}^{+}\right|^{2 p} d x=\left\langle I_{\varepsilon}^{\prime}\left(s_{0} \nu_{0}^{+}+t_{0} v_{0}^{-}\right), s_{0} v_{0}^{+}\right\rangle \\
= & 0 .
\end{aligned}
$$

In the same way, we can obtain that

$$
\begin{aligned}
& \left\langle I_{b_{n}}^{\prime}\left(s_{0} \nu_{0}^{+}+t_{0} \nu_{0}^{-}\right), t_{0} v_{0}^{-}\right\rangle \leq\left\langle I_{\varepsilon}^{\prime}\left(s_{0} v_{0}^{+}+t_{0} v_{0}^{-}\right), t_{0} v_{0}^{-}\right\rangle \\
& \quad=0
\end{aligned}
$$

It follows from Lemma 5 that for all $b_{n} \in[0, \varepsilon]$, there exists a unique pair $\left(s_{n}, t_{n}\right) \in\left(0, s_{0}\right] \times\left(0, t_{0}\right]$ such that $s_{n} \nu_{0}^{+}+t_{n} \nu_{0}^{-} \in$ $\mathscr{M}_{b_{n}}$. Then, for any sequence $\left\{b_{n}\right\}$ with $b_{n} \searrow 0$ as $n \longrightarrow \infty$, we have as $n \longrightarrow \infty$,

$$
\begin{array}{r}
b_{n} s_{n}^{2 p}\left\|\nabla v_{0}^{+}\right\|_{p}^{2 p} \rightarrow 0, \\
b_{n} s_{n}^{p} t_{n}^{p}\left\|\nabla v_{0}^{+}\right\|_{p}^{p}\left\|\nabla v_{0}^{-}\right\|_{p}^{p} \longrightarrow 0, \\
b_{n} t_{n}^{2 p}\left\|\nabla v_{0}^{-}\right\|_{p}^{2 p} \longrightarrow 0 .
\end{array}
$$

According to $\left\langle I_{b_{n}}^{\prime}\left(s_{n} \nu_{0}^{+}+t_{n} \nu_{0}^{-}\right), s_{n} v_{0}^{+}\right\rangle=\left\langle I_{b_{n}}^{\prime}\left(s_{n} v_{0}^{+}+t_{n} v_{0}^{-}\right)\right.$, $\left.t_{n} \nu_{0}^{-}\right\rangle=0$, we have

$$
\begin{aligned}
& a\left\|\nabla v_{0}^{+}\right\|_{p}^{p}+\circ(1)=\lambda \int_{\Omega}\left|v_{0}^{+}\right|^{p} d x+s_{n}^{p} \int_{\Omega}\left|v_{0}^{+}\right|^{2 p} d x \\
& a\left\|\nabla v_{0}^{-}\right\|_{p}^{p}+\circ(1)=\lambda \int_{\Omega}\left|\nu_{0}^{-}\right|^{p} d x+t_{n}^{p} \int_{\Omega}\left|v_{0}^{-}\right|^{2 p} d x .
\end{aligned}
$$

From $\left\langle I_{0}^{\prime}\left(\nu_{0}\right), v_{0}^{ \pm}\right\rangle=0$, we have

$$
\begin{aligned}
& a\left\|\nabla v_{0}^{+}\right\|_{p}^{p}=\lambda \int_{\Omega}\left|v_{0}^{+}\right|^{p} d x+\int_{\Omega}\left|v_{0}^{+}\right|^{2 p} d x \\
& a\left\|\nabla v_{0}^{-}\right\|_{p}^{p}=\lambda \int_{\Omega}\left|\nu_{0}^{-}\right|^{p} d x+\int_{\Omega}\left|\nu_{0}^{-}\right|^{2 p} d x .
\end{aligned}
$$

Combining (74) with (75), we have that as $n \longrightarrow \infty, s_{n} \longrightarrow 1$, $t_{n} \longrightarrow 1$. Lastly, we only need to show $I_{0}\left(u_{0}\right)=I_{0}\left(v_{0}\right)$; then by (68), $u_{0}$ is a ground state sign-changing solution for (11), which changes sign only once. In fact,

$$
\begin{aligned}
I_{0}\left(v_{0}\right) & \leq I_{0}\left(u_{0}\right)=\lim _{n \longrightarrow \infty} I_{b_{n}}\left(u_{b_{n}}\right) \\
& \leq \lim _{n \longrightarrow \infty} I_{b_{n}}\left(s_{n} v_{0}^{+}+t_{n} v_{0}^{-}\right)=I_{0}\left(v_{0}^{+}+v_{0}^{-}\right) \\
& =I_{0}\left(v_{0}\right) .
\end{aligned}
$$

Then, the proof of Theorem 2 is complete.

\section{Data Availability}

The data used to support the findings of this study are included within the article.

\section{Conflicts of Interest}

The authors declare that they have no conflicts of interest regarding the publication of this paper.

\section{Acknowledgments}

This research was supported by the Outstanding Youth Foundation of North University of China (No. JQ201604), the Program for the Innovative Talents of Higher Education Institutions of Shanxi, the Scientific and Technological Innovation Programs of Higher Education Institutions in Shanxi (201802085), and the Natural Science Foundation of Shanxi Province, China (201801D121158).

\section{References}

[1] L. Li and J. Sun, "Existence and multiplicity of solutions for the Kirchhoff equations with asymptotically linear nonlinearities," Nonlinear Analysis: Real World Applications, vol. 26, pp. 391399, 2015.

[2] J. Guo, S. Ma, and G. Zhang, "Solutions of the autonomous Kirchhoff type equations in RN," Applied Mathematics Letters, vol. 82, pp. 14-17, 2018.

[3] Y. Li and L. Li, "Existence and multiplicity of solutions for $\mathrm{p}(\mathrm{x})$-Laplacian equations in RN," Bulletin of the Malaysian Mathematical Sciences Society, vol. 40, no. 4, pp. 1455-1463, 2017.

[4] L. Vilasi, "Eigenvalue estimates for stationary $p(x)$-Kirchhoff problems," Electronic Journal of Differential Equations, vol. 186, pp. 1-9, 2016.

[5] F. Cammaroto and L. Vilasi, "Three solutions for some Dirichlet and Neumann nonlocal problems," Applicable Analysis: An International Journal, vol. 92, no. 8, pp. 1717-1730, 2013.

[6] J. Zhang, Z. Lou, Y. Ji, and W. Shao, "Ground state of Kirchhoff type fractional Schrödinger equations with critical growth," Journal of Mathematical Analysis and Applications, vol. 462, no. 1, pp. 57-83, 2018.

[7] A. Mao, L. Yang, A. Qian, and S. Luan, "Existence and concentration of solutions of Schrödinger-Possion system," Applied Mathematics Letters, vol. 68, pp. 8-12, 2017.

[8] A. Mao and X. Zhu, "Existence and multiplicity results for Kirchhoff problems," Mediterranean Journal of Mathematics, vol. 58, pp. 1-14, 2017. 
[9] Y. Sun, L. Liu, and Y. Wu, "The existence and uniqueness of positive monotone solutions for a class of nonlinear Schrödinger equations on infinite domains," Journal of Computational and Applied Mathematics, vol. 321, pp. 478-486, 2017.

[10] Y. Wang and J. Jiang, "Existence and nonexistence of positive solutions for the fractional coupled system involving generalized p-Laplacian," Advances in Difference Equations, vol. 337, pp. $1-19,2017$.

[11] A. Mao and H. Chang, "Schrödinger-Poisson systems with radial potentials and discontinuous quasilinear nonlinearity," Topological Methods in Nonlinear Analysis, vol. 51, pp. 79-89, 2017.

[12] X. S. Du and A. M. Mao, "Existence and multiplicity of nontrivial solutions for a class of semilinear fractional schrödinger equations," Journal of Function Spaces, vol. 2017, no. 7, Article ID 3793872, 2017.

[13] M. Shao and A. Mao, "Signed and sign-changing solutions of Kirchhoff type problems," Journal of Fixed Point Theory and Applications, vol. 20, no. 2, pp. 1-20, 2018.

[14] J. M. Liu and A. X. Qian, "Ground state solution for a Schrödinger-Poisson equation with critical growth," Nonlinear Analysis: Real World Applications, vol. 40, pp. 428-443, 2018.

[15] J. Liu and Z. Q. Zhao, "Existence of positive solutions to a singular boundary-value problem using variational methods," Electronic Journal of Differential Equations, vol. 135, pp. 1-9, 2014.

[16] X. Hao, H. Wang, L. Liu, and Y. Cui, "Positive solutions for a system of nonlinear fractional nonlocal boundary value problems with parameters and $p$-Laplacian operator," Boundary Value Problems, vol. 182, pp. 1-18, 2017.

[17] A. Mao and S. Luan, "Sign-changing solutions of a class of nonlocal quasilinear elliptic boundary value problems," Journal of Mathematical Analysis and Applications, vol. 383, no. 1, pp. 239-243, 2011.

[18] A. M. Mao, R. A. Jing, S. X. Luan, J. L. Chu, and Y. Kong, "Some nonlocal elliptic problem involving positive parameter," Topological Methods in Nonlinear Analysis, vol. 42, no. 1, pp. 207-220, 2013.

[19] A. Mao and H. Chang, "Kirchhoff type problems in RN with radial potentials and locally Lipschitz functional," Applied Mathematics Letters, vol. 62, pp. 49-54, 2016.

[20] A. M. Mao, Y. Zhu, and S. X. Luan, "Existence of solutions of elliptic boundary value problems with mixed type nonlinearities," Boundary Value Problems, vol. 97, pp. 1-11, 2012.

[21] A. X. Qian, "Sing-changing solutions for nonlinear problems with strong resonance," Electronic Journal of Differential Equations, vol. 17, pp. 1-8, 2012.

[22] A. X. Qian, "Sign-changing solutions for some nonlinear problems with strong resonance," Boundary Value Problems, vol. 2011, no. 1, pp. 1-9, 2011.

[23] A. M. Mao and W. Q. Wang, "Nontrivial solutions of nonlocal fourth order elliptic equation of Kirchhoff type in R3," Journal of Mathematical Analysis and Applications, vol. 1, article no. 459, pp. 556-563, 2018.

[24] M. Shao and A. Mao, "Multiplicity of solutions to SchrödingerPoisson system with concave-convex nonlinearities," Applied Mathematics Letters, vol. 83, pp. 212-218, 2018.

[25] Q. Gao, F. Li, and Y. Wang, "Blow-up of the solution for higherorder Kirchhoff-type equations with nonlinear dissipation," Central European Journal of Mathematics, vol. 9, no. 3, pp. 686698, 2011.
[26] A. Qian, "Infinitely many sign-changing solutions for a Schrödinger equation," Advances in Difference Equations, vol. 2011, article no. 39, pp. 1-6, 2011.

[27] A. M. Mao and Y. S. Chen, "Existence and concentration of solutions for sublinear Schrödinger-Poisson equations," Indian Journal of Pure and Applied Mathematics, vol. 49, no. 2, pp. 339348, 2018.

[28] F. Sun, L. Liu, and Y. Wu, "Infinitely many sign-changing solutions for a class of biharmonic equation with p-Laplacian and Neumann boundary condition," Applied Mathematics Letters, vol. 73, pp. 128-135, 2017.

[29] Y. Sang, "An exact estimate result for a semilinear equation with critical exponent and prescribed singularity," Journal of Mathematical Analysis and Applications, vol. 447, no. 1, pp. 128153, 2017.

[30] J. Zhang, "Standing waves with a critical frequency for nonlinear Schrödinger equations involving critical growth," Applied Mathematics Letters, vol. 63, pp. 53-58, 2017.

[31] W. Shuai, "Sign-changing solutions for a class of Kirchhoff-type problem in bounded domains," Journal of Differential Equations, vol. 259, no. 4, pp. 1256-1274, 2015.

[32] X. H. Tang and B. T. Cheng, "Ground state sign-changing solutions for Kirchhoff type problems in bounded domains," Journal of Differential Equations, vol. 261, no. 4, pp. 2384-2402, 2016.

[33] S. H. Rasouli, H. Fani, and S. Khademloo, "Existence of signchanging solutions for a nonlocal problem of p-Kirchhoff type," Mediterranean Journal of Mathematics, vol. 185, no. 5, pp. 1-14, 2017.

[34] K. J. Brown and Y. Zhang, "The Nehari manifold for a semilinear elliptic equation with a sign-changing weight function," Journal of Differential Equations, vol. 193, no. 2, pp. 481-499, 2003.

[35] M. Willem, Minimax Theorems, Birkhäuser, Boston, Mass, USA, 1996. 


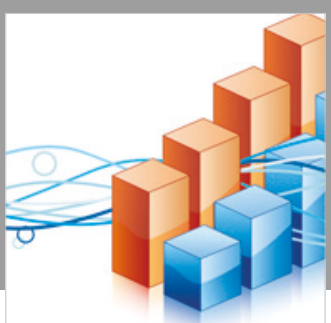

Advances in

Operations Research

\section{-n-m}
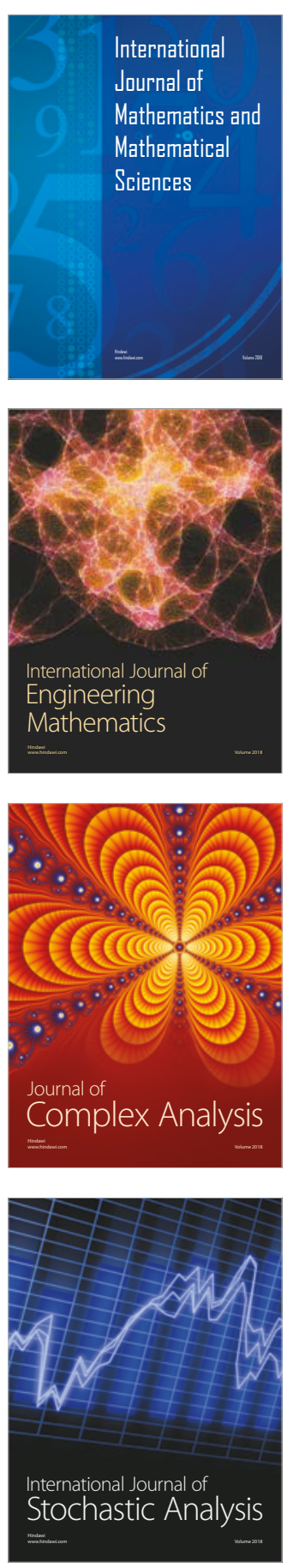
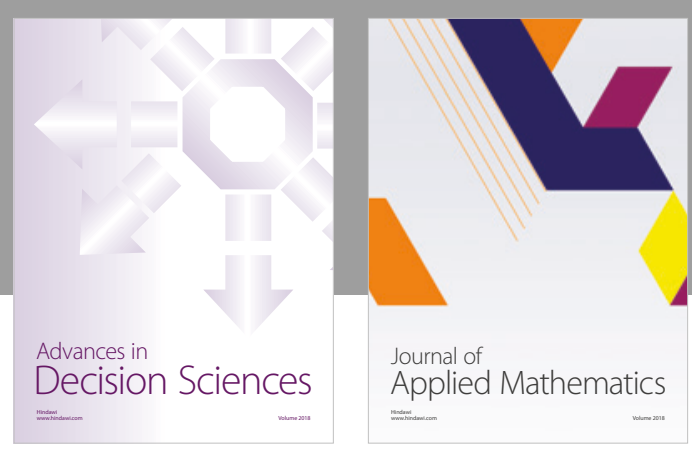

Journal of

Applied Mathematics
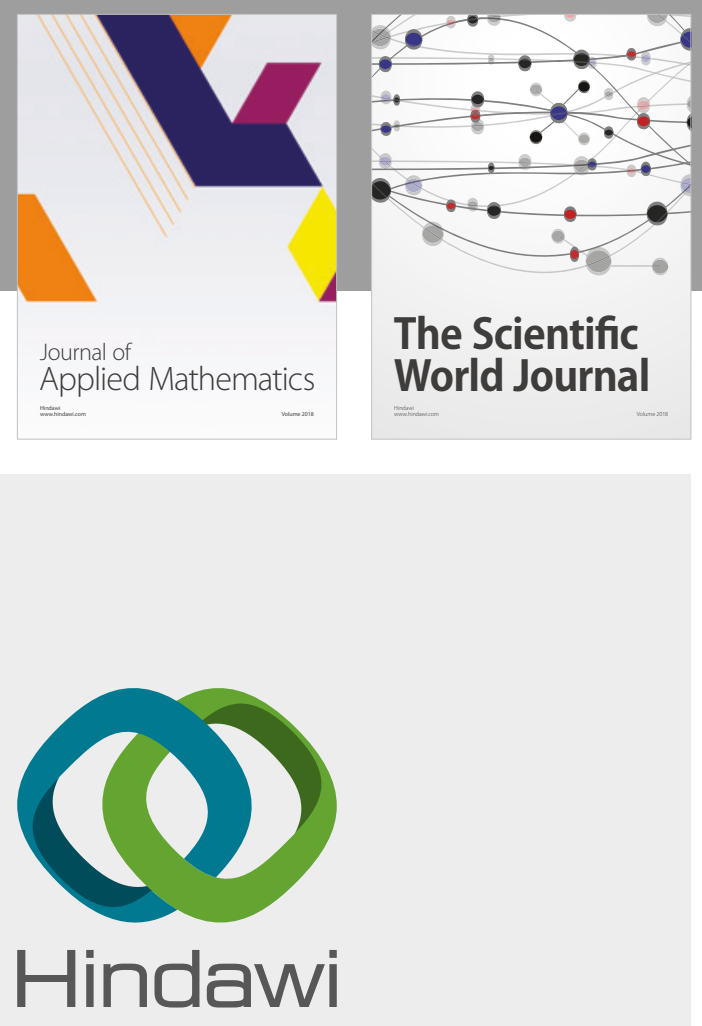

Submit your manuscripts at

www.hindawi.com

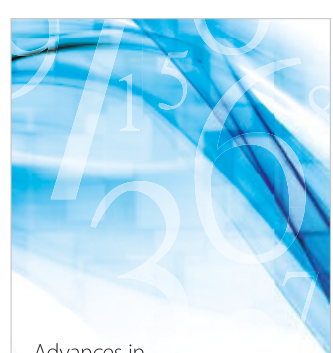

Advances in
Numerical Analysis
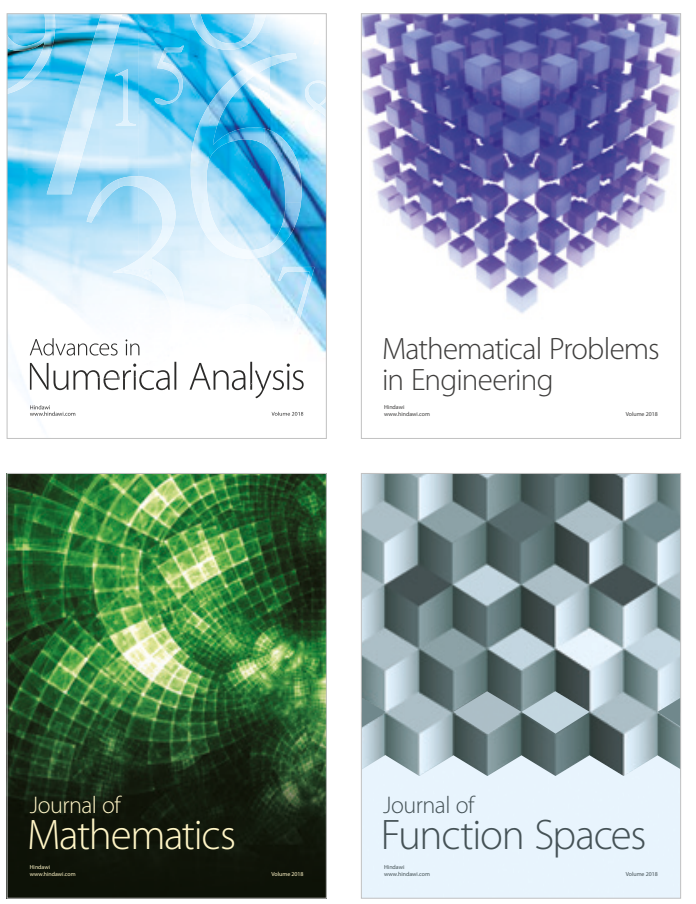

Mathematical Problems in Engineering

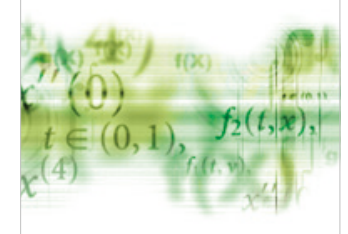

International Journal of

Differential Equations



Journal of

Function Spaces
The Scientific

World Journal

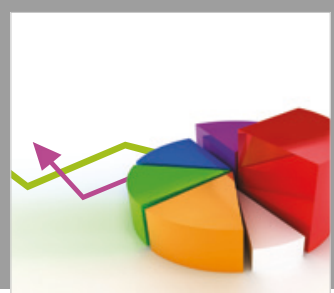

Journal of

Probability and Statistics
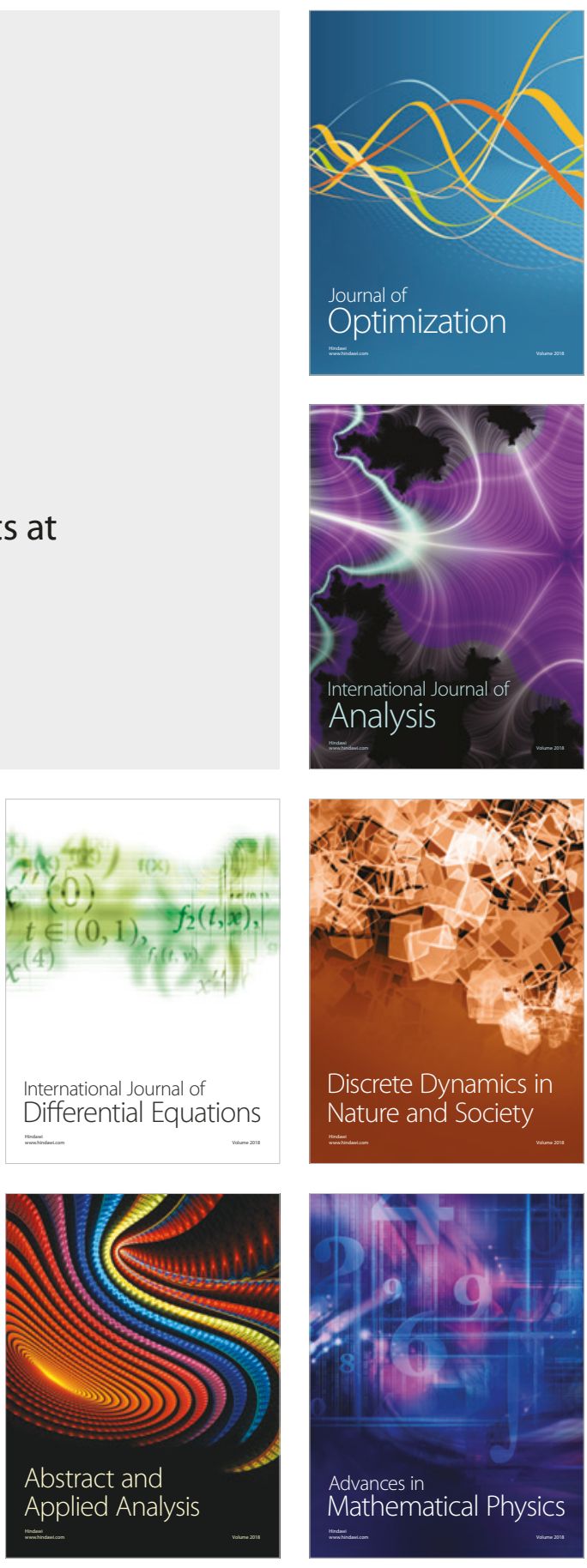